\title{
Factores asociados a dehiscencia de anastomosis intestinal
}

\section{Factors associated with dehiscence of intestinal anastomosis}

\author{
Andrea S. Álvarez-Villaseñor ${ }^{1}$, Silvia del Carmen Prado-Rico², Jorge I. Morales-Alvarado', \\ Laura L. Reyes-Aguirre ${ }^{3}$, Clotilde Fuentes-Orozco ${ }^{3}$ y Alejandro González-Ojeda ${ }^{3 *}$ \\ ${ }^{1}$ Departamento de Cirugía General, Benemérito Hospital General Juan María de Salvatierra, La Paz, Baja California Sur; ${ }^{2}$ Departamento de Cirugía \\ Plástica, Estética y Reconstructiva, Hospital Rubén Leñero, Ciudad de México; ${ }^{3}$ Unidad de Investigación Biomédica 02, Hospital de Especialidades, \\ Unidad Médica de Alta Especialidad, Centro Médico Nacional de Occidente, Instituto Mexicano del Seguro Social, Guadalajara, Jalisco. México
}

\section{Resumen}

Objetivo: Identificar los factores asociados a dehiscencia de anastomosis de intestino delgado y grueso. Método: Se incluyeron 92 anastomosis de intestino delgado y grueso, en mayores de 18 años, realizadas en 2012-2016. Se evaluaron factores asociados en el preoperatorio, el transoperatorio y el posoperatorio. Resultados: Se presentó dehiscencia de anastomosis en el $13 \%$ de los casos. Se encontró una asociación significativa con ingesta previa de medicamentos $(p=0.05 ;$ odds ratio [OR]: 1.17; IC 95\%) y con anastomosis primaria ( $p=0.05$; OR: 3.6; 0.92-14.5). En los pacientes con dehiscencia se incrementó la estancia intrahospitalaria. Conclusión: La presencia de dehiscencia de anastomosis fue similar a lo reportado en la literatura. Los factores asociados fueron la ingesta previa de medicamentos y la anastomosis primaria.

Palabras clave: Anastomosis intestinal. Dehiscencia. Fuga anastomótica.

\begin{abstract}
Objective: To identify the factors associated with dehiscence of anastomosis of the small and large intestine. Method: 92 anastomoses of the small and large intestine were included in patients over 18 years of age, performed in 2012-2016. Associated factors were evaluated in pre, trans and postoperative. Results: Anastomosis dehiscence was presented in $13 \%$. A significant association was found for previous medication intake ( $p=0.05$; odds ratio [OR]: 1.17; 1.024-1.33) and primary anastomosis ( $p=0.05$, OR: 3.6; 0.92-14.5). In patients with dehiscence, the hospital stay was increased. Conclusion: The presence of dehiscence of anastomosis was similar to that reported in the literature. The associated factors were previous medication intake, and primary anastomosis.
\end{abstract}

Key words: Intestinal anastomosis. Dehiscence. Anastomotic leak.

\section{Introducción}

La dehiscencia de anastomosis es una de las complicaciones quirúrgicas más temidas en el ámbito de la cirugía digestiva ${ }^{1-8}$ y se asocia con un aumento de la morbimortalidad y de la estancia hospitalaria'. La frecuencia y la gravedad de las complicaciones varían de acuerdo con el sitio donde se presentan. Actualmente

\section{Correspondencia:}

*Alejandro González-Ojeda

Belisario Domínguez, 100

Col. Independencia

C.P 44340., Guadalajara, Jal., México

E-mail: avygail5@gmail.com
Fecha de recepción: 07-01-2020

Cir Cir. 2021;89(2):233-242

Fecha de aceptación: 05-06-2020

DOI: $10.24875 / C I R U .20000018$
Contents available at PubMed

www.cirugiaycirujanos.com

0009-7411/C 2020 Academia Mexicana de Cirugía. Publicado por Permanyer. Este es un artículo open access bajo la licencia CC BY-NC-ND (http://creativecommons.org/licenses/by-nc-nd/4.0/). 
se considera un indicador para la calidad en la atención y los cuidados quirúrgicos, que a su vez funge como punto de corte para la evaluación de los protocolos y los estándares de diferentes unidades hospitalarias, mismos que pueden ser comparados entre sít0-12.

Se han identificado numerosos factores de riesgo que pueden condicionar la aparición de la dehiscencia de una anastomosis intestinal. Con fines de estudio, podríamos agruparlos en tres tipos: aquellos plenamente identificados, algunos aún en estudio y finalmente los que se consideraban con efecto deletéreo o protector y que en estudios recientes han demostrado un efecto contrario. Los porcentajes de dehiscencia varían según las series del 0.3 al $5.5 \%$ en el intestino delgado hasta el $21 \%$ en el intestino grueso ${ }^{12}$.

En los últimos años, diversos estudios coinciden en la búsqueda de factores relacionados con la presencia de dehiscencia y hacen hincapié en los hallazgos clínicos, los antecedentes nutricionales y las pruebas laboratorio o de imagen que orientan a la detección temprana de esta complicación, ya que la presencia de tres o más factores puede incrementar la probabilidad de dehiscencia hasta en un $50 \%$ 4,5,13. La mortalidad en los pacientes con dehiscencia de anastomosis va del 0.5 al $15 \%{ }^{13}$.

Morse, et al..$^{12}$ determinaron los factores de riesgo para dehiscencia más comunes en 682 anastomosis: enfermedad pulmonar (29\%), albúmina previa $<3.0 \mathrm{mg} / \mathrm{dl}$ (37\%), peritonitis preoperatoria (34\%), tensión en la anastomosis $(5 \%)$, transfusiones preoperatorias (15.8\%), presencia de cáncer (19.4\%) y colocación de drenajes (3\%); y como factores predictores, en el análisis multivariado, solo la tensión de la anastomosis (odds ratio [OR]: 10.1; intervalo de confianza del 95\% [IC95\%]: 1.4-75.9), el uso de drenajes (OR: 8.9; IC95\%: 4.4-18.4) y la transfusión sanguínea (OR: 4.2; IC95\%: 1.4-12.3).

La dificultad para predecir la ocurrencia de una dehiscencia radica en la identificación de los factores asociados con esta complicación. En la práctica, la dehiscencia sucede incluso en pacientes jóvenes, bien nutridos, sin condiciones comórbidas y a pesar de una técnica quirúrgica satisfactoria, por lo que es importante establecer los factores asociados a la dehiscencia de las anastomosis en cada población $\mathrm{n}^{13}$.

El Hospital General Juan María de Salvatierra es un centro hospitalario de referencia ubicado en el Estado de Baja California Sur, que atiende a pacientes de nivel socioeconómico bajo y sin seguridad social, con diversas patologías abdominales, entre las cuales al menos 25 casos por año requerirán resección y anastomosis, por lo que el objetivo de este estudio fue identificar la presencia de factores asociados a dehiscencia de anastomosis de intestino delgado y grueso en nuestro nosocomio, con la finalidad de implementar medidas preventivas para evitar la aparición de este evento.

\section{Método}

Se realizó un estudio observacional descriptivo en el que se registraron 92 pacientes a los que se realizó una anastomosis de intestino delgado y grueso, en los años 2012-2016, mediante muestreo no probabilístico de casos consecutivos. Se incluyeron pacientes mayores de 18 años a los que se realizó anastomosis manual o mecánica, intraperitoneal 0 extraperitoneal, electiva o urgente, de intestino delgado o grueso, y con valoración preoperatoria ASA I-III. La principal fuente de obtención de datos fueron los expedientes clínicos que tuvieran diagnósticos estandarizados de acuerdo con el código internacional de enfermedades (CIE-10) como Z933 (colostomía), Z433 (atención de colostomía), K914 (disfunción de colostomía o enterostomía), Z432 (atención de ileostomía), Z932 (ileostomía), Z465 (prueba y ajuste de ileostomía o de otro dispositivo intestinal), Z980 (estado de derivación intestinal o anastomosis), Y832 (operación quirúrgica con anastomosis, derivación o injerto) o Y652 (falla en la sutura o ligadura durante la operación quirúrgica).

Las variables se clasificaron en:

- Preoperatorias: sexo, edad, toxicomanías, ingesta previa de medicamentos, comorbilidad, estado nutricional según el índice de masa corporal (IMC, < 18 desnutrido, 18.1-24.9 peso normal, 25-29.9 sobrepeso, $>30$ obesidad), albúmina sérica $(\mathrm{mg} / \mathrm{dl})$, profilaxis antibiótica, preparación intestinal, estado físico del asa y tipo de cirugía.

- Transoperatorias: sepsis abdominal preoperatoria, duración de la cirugía (minutos), colocación de drenajes, transfusión transoperatoria, causa de la cirugía, anastomosis primaria, tipo de anastomosis y estoma protector o profiláctico.

- Posoperatorias: dehiscencia de anastomosis intestinal, inicio de dieta enteral (vías), uso de nutrición parenteral total, estancia en la unidad de cuidados intensivos, estancia hospitalaria y mortalidad.

Se realizó estadística descriptiva mediante promedios y desviaciones estándar, frecuencias y porcentajes. El análisis inferencial de las variables cualitativas se llevó 
a cabo con la prueba de ji al cuadrado o con la prueba exacta de Fisher; para la variables cuantitativas se utilizó la prueba t de Student o la prueba U de Mann-Whitney. Se realizaron cálculos para factores de riesgo (OR). Se usó el paquete estadístico SPSS v21.

El presente estudio fue aprobado por el comité de investigación de la unidad hospitalaria con el número de registro 004-004-2016.

\section{Resultados}

Se estudiaron 92 pacientes con una media de edad de $45.5 \pm 16.3$ años. Se realizaron 70 (76\%) anastomosis en pacientes varones. Tenían alguna comorbilidad 28 (30.4\%) pacientes, y más del 50\% cursaban con sobrepeso y obesidad. Las cirugías realizadas fueron electivas en 67 (72.8\%) casos (Tabla 1).

El tiempo promedio del evento quirúrgico fue de 175.5 minutos. La evidencia de sepsis abdominal se presentó en $25(27.2 \%)$ pacientes. Requirieron transfusión de hemoderivados 19 (21\%) pacientes. La anastomosis colónica mostró predominio sobre la ileal (64 [69.6\%]). Solo en el 25\% de los casos se colocó drenaje. En 67 (72.8\%) pacientes, las anastomosis se realizaron por un diagnóstico benigno (restitución de tránsito intestinal por estomas previos, oclusión intestinal, etc.). La técnica mayormente utilizada fue la manual, en 88 (95.7\%) casos (Tabla 2). El promedio del tiempo trascurrido entre la primera cirugía y la restitución del tránsito intestinal en los casos con colostomía o ileostomía previa fue de 5 meses.

El $13 \%$ de las anastomosis presentaron fuga, con un promedio de días hasta la identificación del evento de $6(6.5 \pm 3.5)$. La mediana de la estancia hospitalaria fue de $13.6 \pm 15.8$ días. Ingresaron a la unidad de cuidados intensivos 9 (9.8\%) pacientes. Requirieron el inició de nutrición parenteral total 21 (23\%) pacientes. La mortalidad global fue de 7 (7.6\%) casos. La mortalidad asociada en los pacientes sin fuga de anastomosis fue por trauma de cráneo grave, infarto agudo al miocardio y tromboembolia pulmonar. En los pacientes con dehiscencia solo ocurrió una defunción (Tabla 3).

Para la variable ingesta previa de medicamentos, los fármacos más comunes fueron antiinflamatorios no esteroideos (AINE), metformina, enalapril y pravastatina, en este orden. Se encontró una asociación estadísticamente significativa entre la presencia o no de dehiscencia de anastomosis y la ingesta previa de medicamentos ( $p=0.05$; OR: 1.17 ; IC 95\%); el resto de las variables preoperatorias no mostraron diferencia
Tabla 1. Frecuencias, características generales de los pacientes en el preoperatorio $(n=92)$

\begin{tabular}{|c|c|c|c|c|c|}
\hline \multicolumn{2}{|l|}{ Variable } & $\begin{array}{l}\text { Frecuencia } \\
\text { (n) }\end{array}$ & $\begin{array}{l}\text { Porcentaje } \\
(\%)\end{array}$ & Media & $\mathrm{DE}$ \\
\hline \multicolumn{2}{|l|}{ Edad } & & & 45.5 & \pm 16.3 \\
\hline \multicolumn{2}{|c|}{ Niveles de albumina sérica } & & & 3.8 & \pm 0.91 \\
\hline \multirow[t]{2}{*}{ Sexo } & Masculino & 70 & 76.1 & & \\
\hline & Femenino & 22 & 23.9 & & \\
\hline \multirow[t]{2}{*}{ Toxicomanías } & $\mathrm{Si}$ & 61 & 66.3 & & \\
\hline & No & 31 & 33.7 & & \\
\hline \multirow{2}{*}{$\begin{array}{l}\text { Ingesta } \\
\text { previa de } \\
\text { medicamentos }\end{array}$} & $\mathrm{Si}$ & 29 & 31.5 & & \\
\hline & No & 63 & 68.5 & & \\
\hline \multirow[t]{2}{*}{ Comorbilidades } & $\mathrm{Si}$ & 28 & 30.4 & & \\
\hline & No & 64 & 69.6 & & \\
\hline \multirow[t]{4}{*}{ IMC } & Desnutrido & 4 & 4.3 & & \\
\hline & Normal & 37 & 40.2 & & \\
\hline & Sobrepeso & 32 & 34.8 & & \\
\hline & Obesidad & 19 & 20.7 & & \\
\hline \multirow{2}{*}{$\begin{array}{l}\text { Preparación } \\
\text { intestinal }\end{array}$} & $\mathrm{Si}$ & 33 & 35.9 & & \\
\hline & No & 59 & 64.1 & & \\
\hline \multirow{2}{*}{$\begin{array}{l}\text { Profilaxis } \\
\text { antibiótica }\end{array}$} & $\mathrm{Si}$ & 40 & 43.5 & & \\
\hline & No & 52 & 56.5 & & \\
\hline \multirow{3}{*}{$\begin{array}{l}\text { Estado físico } \\
\text { de asa }\end{array}$} & I & 29 & 31.5 & & \\
\hline & $\|$ & 43 & 46.7 & & \\
\hline & III & 20 & 21.7 & & \\
\hline \multirow[t]{2}{*}{ Tipo de cirugía } & Electiva & 67 & 72.8 & & \\
\hline & Urgencia & 25 & 27.2 & & \\
\hline
\end{tabular}

(sexo, tabaquismo, comorbilidad, IMC, preparación intestinal, profilaxis antibiótica, estado físico ASA y tipo de cirugía). Para las variables transoperatorias, sí se encontró asociación significativa en la realización de anastomosis primaria ( $p=0.05$; OR: $3.6 ; 0.92-14.5$ ), pero en el resto de las variables no hubo diferencia significativa (sepsis abdominal, transfusiones, sitio anatómico de la anastomosis, colocación de drenajes, causa de la cirugía, tipo de anastomosis y estoma protector). En las variables posoperatorias, tales como 
Tabla 2. Frecuencias, características generales de los pacientes en el tras-operatorio $(n=92)$

\begin{tabular}{|c|c|c|c|c|c|}
\hline \multicolumn{2}{|l|}{ Variable } & Frecuencia & Porcentaje & Media & DE \\
\hline \multicolumn{2}{|l|}{$\begin{array}{l}\text { Duración de la } \\
\text { cirugía (minutos) }\end{array}$} & & & 175.5 & 65 \\
\hline \multirow{2}{*}{$\begin{array}{l}\text { Sepsis } \\
\text { abdominal }\end{array}$} & $\mathrm{Si}$ & 25 & 27.2 & & \\
\hline & No & 67 & 72.8 & & \\
\hline \multirow[t]{5}{*}{$\begin{array}{l}\text { Transfusiones } \\
\text { transoperatoria }\end{array}$} & $\begin{array}{l}\text { Sin } \\
\text { trasfusión }\end{array}$ & 79 & 79.3 & & \\
\hline & 1 & 10 & 10.9 & & \\
\hline & 2 & 7 & 7.6 & & \\
\hline & 3 & 1 & 1.1 & & \\
\hline & 5 & 1 & 1.1 & & \\
\hline \multirow{2}{*}{$\begin{array}{l}\text { Sitio } \\
\text { anatómico de } \\
\text { la anastomosis }\end{array}$} & $\begin{array}{l}\text { Intestino } \\
\text { delgado }\end{array}$ & 28 & 30.4 & & \\
\hline & $\begin{array}{l}\text { Intestino } \\
\text { grueso }\end{array}$ & 64 & 69.6 & & \\
\hline \multirow{2}{*}{$\begin{array}{l}\text { Colocación de } \\
\text { drenajes }\end{array}$} & $\mathrm{Si}$ & 69 & $75 \%$ & & \\
\hline & No & 23 & $25 \%$ & & \\
\hline \multirow{3}{*}{$\begin{array}{l}\text { Causa de la } \\
\text { cirugía }\end{array}$} & Benigna & 67 & 72.8 & & \\
\hline & Maligna & 16 & 17.4 & & \\
\hline & Traumatismo & 9 & 9.8 & & \\
\hline \multirow{2}{*}{$\begin{array}{l}\text { Anastomosis } \\
\text { primaria }\end{array}$} & $\mathrm{Si}$ & 45 & 48.9 & & \\
\hline & No & 47 & 51.1 & & \\
\hline \multirow{2}{*}{$\begin{array}{l}\text { Tipo de } \\
\text { Anastomosis }\end{array}$} & Manual & 88 & 95.7 & & \\
\hline & Mecánica & 4 & 4.3 & & \\
\hline \multirow{2}{*}{$\begin{array}{l}\text { Estoma } \\
\text { protector o } \\
\text { profiláctico }\end{array}$} & si & 12 & 13 & & \\
\hline & no & 80 & 87 & & \\
\hline
\end{tabular}

DE: desviación estándar.

uso de nutrición parenteral total, pase a unidad de cuidados intensivos o mortalidad asociada al procedimiento, no se evidenció diferencia significativa (Tabla 4). Los días de estancia intrahospitalaria se incrementaron en los pacientes con dehiscencia $(p=0.000 ; 8.8-26.9)$. No se encontró diferencia en las cifras de albumina, la edad, la duración de la cirugía ni el inicio de la dieta enteral (Tabla 5).

Al analizar el mismo orden de variables, preoperatorias, transoperatorias y posoperatorias, pero según el sitio anatómico de la anastomosis (intestino delgado o intestino grueso), las variables que mostraron diferencia significativa en el preoperatorio fueron la ingesta previa de medicamentos $(p=0.005$; OR: 1.3;
Tabla 3. Frecuencias, características generales de los pacientes en el posoperatorio $(n=92)$

\begin{tabular}{|c|c|c|c|c|c|}
\hline \multicolumn{2}{|l|}{ Variables } & $\begin{array}{c}\text { Frecuencia } \\
\text { (n) }\end{array}$ & $\begin{array}{c}\text { Porcentaje } \\
(\%)\end{array}$ & Media & DE \\
\hline \multicolumn{2}{|l|}{$\begin{array}{l}\text { Estancia } \\
\text { hospitalaria (días) }\end{array}$} & & & 13.6 & 15.8 \\
\hline \multicolumn{2}{|l|}{$\begin{array}{l}{ }^{*} \text { Inicio de Dieta } \\
\text { enteral }\end{array}$} & & & 3.7 & 2.2 \\
\hline \multicolumn{2}{|l|}{$\begin{array}{l}{ }^{* *} \text { identificación } \\
\text { de la fuga }\end{array}$} & & & 6.5 & 3.5 \\
\hline \multirow{2}{*}{$\begin{array}{l}\text { Dehiscencia de } \\
\text { anastomosis } \\
\text { intestinal }\end{array}$} & $\mathrm{Si}$ & 12 & 13 & & \\
\hline & No & 80 & 87 & & \\
\hline \multirow[t]{2}{*}{ Uso de NPT } & $\mathrm{Si}$ & 21 & 22.8 & & \\
\hline & No & 71 & 77.2 & & \\
\hline \multirow{2}{*}{$\begin{array}{l}\text { Pase a Unidad } \\
\text { de cuidados } \\
\text { intensivos }\end{array}$} & $\mathrm{Si}$ & 9 & 9.8 & & \\
\hline & No & 83 & 90.2 & & \\
\hline \multirow[t]{2}{*}{ Mortalidad global } & $\mathrm{Si}$ & 7 & 7.6 & & \\
\hline & No & 85 & 92.4 & & \\
\hline \multirow{2}{*}{$\begin{array}{l}\text { Mortalidad } \\
\text { asociada al } \\
\text { procedimiento. }\end{array}$} & $\mathrm{Si}$ & 1 & 8.3 & & \\
\hline & No & 11 & 91.7 & & \\
\hline
\end{tabular}

DE: desviación estándar; NPT: nutrición parenteral total.

1.05-1.67), el tipo de cirugía y la cirugía electiva vs. de urgencia ( $p=0.006$; OR: 1.6; 1.05-2.48); en el transoperatorio, la presencia de sepsis abdominal $(p=0.006$; OR: $3.7 ; 1.42-9.93)$. En las variables posoperatorias, se encontró asociación significativa para el uso de nutrición parenteral total $(p=0.04$; OR: 2.6; 0.97-7.3) (Tabla 6).

Se realizó manejo quirúrgico de la dehiscencia en el $50 \%$ de los pacientes; el resto recibió tratamiento conservador.

\section{Discusión}

La dehiscencia de anastomosis en esta serie fue del $13 \%$ en el total de los casos; aunque se encuentra dentro de lo reportado, en otros centros la incidencia es menor del $6 \%{ }^{14}$.

Matsuda, et al. ${ }^{1}$, en un ensayo clínico controlado, compararon anastomosis de intestino delgado antiperistálticas contra isoperistálticas, sin encontrar evidencia de dehiscencia atribuida a una u otra técnica, y en aquellos pacientes que la presentaron se atribuyó a sepsis localizada, inflamación de la anastomosis 
o cambios de presión en el asa, además de a la respuesta de cada paciente al evento quirúrgico.

Alves, et al. ${ }^{11}$ identificaron como factores de riesgo independientes la presencia de leucocitosis preoperatoria $>10.000 / \mathrm{mm}^{3}$ (OR: 2.3), sepsis intraabdominal preoperatoria (OR: 4.9 ), dificultades durante la anastomosis (OR: 10.3), anastomosis colocolónica (OR: 6.24) y hemotransfusiones en el posoperatorio (OR: 4.59).

Morse, et al..$^{12}$ analizaron 682 pacientes sometidos a anastomosis intestinal y encontraron 38 con dehiscencia, entre los cuales identificaron una asociación con los siguientes factores: antecedente de enfermedad pulmonar (por la probable hipoperfusión relacionada), desnutrición, hemotransfusiones en el perioperatorio, sangrado transoperatorio, infección intraabdominal, sitio de la fuga anastomótica, tensión de la anastomosis y uso de drenajes.

En un estudio realizado por Muñoz, et al..$^{14}$ en Chile con 748 pacientes se encontró una fuerte asociación de la hipoalbuminemia y las transfusiones como factores de riesgo para el desarrollo de dehiscencias. En nuestra serie de casos se demostró que realizar anastomosis primaria y la ingesta previa de medicamentos, tales como AINE, quimioterapia, esteroides y anticoagulantes, pueden condicionar de manera significativa la aparición de dehiscencias. Existen estudios de intervención en los que la toma de decisión clínica influye en la disminución de las dehiscencias de anastomosis de un $10 \%$ a un $4.5 \%$, tales como reducir el uso de AINE los 5 días previos y hasta los 7 días posteriores a la cirugía, el manejo de líquidos intravenosos y evitar las anastomosis primarias en las resecciones de urgencia ${ }^{15}$.

Un factor que no fue posible comparar con los hallazgos reportados en la literatura fue el uso de engrapadoras, puesto que en esta serie dicha técnica solo fue posible en cuatro pacientes, por la limitación del acceso a los insumos con que cuenta la unidad hospitalaria. Aun cuando no hay un método de referencia para la técnica de anastomosis intestinal, manual o con engrapadora, existen estudios, como el realizado por Kosuge, et al. ${ }^{16}$ con 684 anastomosis intestinales en Japón, que describen que la técnica más segura para realizar una anastomosis en cirugía de colon, con evidencia de una menor incidencia de fuga, es la anastomosis terminoterminal con engrapadora intestinal (anastomosis de triangulación).

La preparación intestinal se realizó en menos de la mitad de los pacientes sometidos a cirugía colorrectal, y el tipo de anastomosis utilizada fue únicamente mecánica. Existen controversias entre los protocolos de preparación mecánica del colon reportados desde el año 2000, ya que emiten diferentes opiniones sobre la implementación o no de esta, siendo más predominante su contraindicación; sin embargo, dichas aseveraciones se han ido refutando con nuevas evidencias que, aunque se postulan con un nivel de evidencia bajo-moderado, hablan de los beneficios otorgados al paciente con la preparación mecánica del colon y la administración de antibióticos orales en cirugía colorrectal, sobre todo en la prevención de la infección del sitio quirúrgico, y la incidencia baja en prevención de fugas anastomóticas ${ }^{17}$.

A pesar de que no se encontró una asociación entre la dehiscencia y la presencia de drenajes, estos se colocaron en el $83 \%$ de los pacientes que presentaron dehiscencia de anastomosis. Un metaanálisis realizado en el año 2018 basado en cuatro ensayos clínicos (con un total de 766 pacientes) demostró la poca utilidad de los drenajes intraabdominales en presencia de anastomosis intestinales ${ }^{18}$, razón por la cual se debe tener precaución al tomar la decisión de colocarlos.

No ha sido posible demostrar la relación entre el tiempo transcurrido desde la primera cirugía y la restitución del tránsito intestinal como factor de riesgo para complicaciones, principalmente en relación con las infecciones del sitio quirúrgico y las fugas anastomóticas. Por otro lado, existe evidencia que sustenta que la estancia hospitalaria prolongada y el tabaquismo previo son factores predisponentes.

En nuestra serie, el promedio de restitución fue de 5 meses, mientras que para Hallam, et al. ${ }^{19}$, en el Reino Unido, en 288 cirugías, el promedio fue de 11 meses. De acuerdo con el momento de identificación de los signos o síntomas de fuga anastomótica por el cirujano en la misma hospitalización, es decir, en el posoperatorio inmediato y mediato, el $50 \%$ de los pacientes incluidos en nuestro estudio recibieron manejo con tratamiento conservador, decidido por el médico tratante con vigilancia intrahospitalaria y control radiográfico, a razón de un gasto menor de $100 \mathrm{ml}$ por día; el resto requirieron laparotomía para control de la fuga y estoma. Este manejo sigue siendo la conducta de elección recomendada por las clínicas de cirugía de colon y recto, ya que a pesar de las múltiples alternativas no quirúrgicas para el manejo de este tipo de pacientes (drenaje percutáneo, antibióticos, terapia Vaccum Assisted Closure [VAC], stents intestinales) la reintervención desempeña un papel fundamental en el control de la fuga, sobre todo en aquellos pacientes que no mejoran con el tratamiento conservador, como en caso de presencia de sepsis y peritonitis persistente, ya que es 
Cirugía y Cirujanos. 2021;89(2)

Tabla 4. Análisis univariado asociado a la presencia de dehiscencia de anastomosis $(n=92)$

\begin{tabular}{|c|c|c|c|c|c|}
\hline \multicolumn{3}{|c|}{ Variables cualitativas } & \multicolumn{2}{|c|}{ Dehiscencia de anastomosis } & \multirow{2}{*}{$\begin{array}{c}p^{*} \\
(\text { OR; IC95\%) }\end{array}$} \\
\hline & & & Sí $(n=12)$ & No $(n=80)$ & \\
\hline \multirow[t]{21}{*}{ Preoperatorio } & \multirow[t]{2}{*}{ Sexo } & Masculino & $9(9.8 \%)$ & $61(66.3 \%)$ & \multirow{2}{*}{$\begin{array}{c}0.58 \\
(0.934 ; 0.022-3.80)\end{array}$} \\
\hline & & Femenino & $3(3.3 \%)$ & $19(20.7 \%)$ & \\
\hline & \multirow[t]{2}{*}{ Tabaquismo } & Sí & $9(9.8 \%)$ & $52(56.5 \%)$ & \multirow{2}{*}{$\begin{array}{c}0.37 \\
(1.6 ; 0.40-6.45)\end{array}$} \\
\hline & & No & $3(3.3 \%)$ & $28(30.4 \%)$ & \\
\hline & \multirow[t]{2}{*}{ Ingesta previa de medicamentos† } & Sí & $1(1.1 \%)$ & $28(30.4 \%)$ & \multirow{2}{*}{$\begin{array}{c}0.05 \\
(1.17 ; 1.024-1.33)\end{array}$} \\
\hline & & No & $11(12.0 \%)$ & $52(56.5 \%)$ & \\
\hline & \multirow[t]{2}{*}{ Comorbilidad } & Sí & $1(1.1 \%)$ & $27(29.3 \%)$ & \multirow{2}{*}{$\begin{array}{c}0.06 \\
(0.178 ; 0.022-1.45)\end{array}$} \\
\hline & & No & $11(12.0 \%)$ & $53(57.6 \%)$ & \\
\hline & \multirow[t]{4}{*}{ IMC } & Desnutrido & $1(1.1 \%)$ & $3(3.3 \%)$ & \multirow{4}{*}{$\begin{array}{c}0.511 \\
(1.21 ; 0.40-0.61)\end{array}$} \\
\hline & & Normal & $4(4.3 \%)$ & $33(35.9 \%)$ & \\
\hline & & Sobrepeso & $4(4.3 \%)$ & $28(30.4 \%)$ & \\
\hline & & Obesidad & $3(3.3 \%)$ & $16(17.4 \%)$ & \\
\hline & \multirow[t]{2}{*}{ Preparación intestinal } & Sí & $3(3.3 \%)$ & $30(32.6 \%)$ & \multirow{2}{*}{$\begin{array}{c}0.30 \\
(0.55 ; 0.139-2.21)\end{array}$} \\
\hline & & No & $9(9.8 \%)$ & $50(50.4 \%)$ & \\
\hline & \multirow[t]{2}{*}{ Profilaxis antibiótica } & Sí & $5(5.4 \%)$ & $35(38.0 \%)$ & \multirow{2}{*}{$\begin{array}{c}0.57 \\
(0.918 ; 0.26-3.14)\end{array}$} \\
\hline & & No & $7(7.6 \%)$ & $45(48.9 \%)$ & \\
\hline & \multirow[t]{3}{*}{ Estado físico ASA } & 1 & $6(6.5 \%)$ & $23(25.0 \%)$ & \multirow{3}{*}{$\begin{array}{c}0.152 \\
(0.11 ; 0.07-0.22)\end{array}$} \\
\hline & & $\|$ & $4(4.3 \%)$ & $39(42.4 \%)$ & \\
\hline & & III & $2(2.2 \%)$ & $18(19.6 \%)$ & \\
\hline & \multirow[t]{2}{*}{ Tipo de cirugía } & Electiva & $7(7.6 \%)$ & $60(65.2 \%)$ & \multirow{2}{*}{$\begin{array}{c}0.19 \\
(0.467 ; 0.133-1.63)\end{array}$} \\
\hline & & Urgencia & $5(5.4 \%)$ & $20(21.7 \%)$ & \\
\hline \multirow[t]{14}{*}{ Transoperatorio } & \multirow[t]{2}{*}{ Sepsis abdominal } & Sí & $5(5.4 \%)$ & $20(21.7 \%)$ & \multirow{2}{*}{$\begin{array}{c}0.12 \\
(2.1 ; 0.611-7.5)\end{array}$} \\
\hline & & No & $7(7.6 \%)$ & $60(65.2 \%)$ & \\
\hline & \multirow[t]{5}{*}{ Transfusiones transoperatorias } & Ninguna & $10(10.8 \%)$ & $63(68.4 \%)$ & \multirow{5}{*}{$\begin{array}{c}0.42 \\
(2.1 ; 0.30-0.50)\end{array}$} \\
\hline & & 1 & $1(1.1 \%)$ & $9(9.7 \%)$ & \\
\hline & & 2 & $1(1.1 \%)$ & $6(6.5 \%)$ & \\
\hline & & 3 & $0(0 \%)$ & $1(1.1 \%)$ & \\
\hline & & 5 & $0(0 \%)$ & $1(1.1 \%)$ & \\
\hline & \multirow[t]{2}{*}{ Sitio anatómico de la anastomosis } & Intestino delgado & $1(1.1 \%)$ & $27(29.3 \%)$ & 0.06 \\
\hline & & Intestino grueso & $11(12 \%)$ & $53(57.6 \%)$ & (U1r;0.2 \\
\hline & Drenajes & Sí & $10(10.9 \%)$ & $59(64.1 \%)$ & 0.23 \\
\hline & & No & $2(2.2 \%)$ & $21(22.8 \%)$ & $(1.10,0.00-0.19)$ \\
\hline & Causa de la cirugía & Benigna & $10(10.9 \%)$ & $57(62 \%)$ & 0.39 \\
\hline & & Maligna & $1(10.1 \%)$ & $15(16.3 \%)$ & $(1.1,0 . \angle 9-0.49)$ \\
\hline & & Traumatismo & $1(1.1 \%)$ & $8(8.7 \%)$ & \\
\hline
\end{tabular}


Tabla 4. Análisis univariado asociado a la presencia de dehiscencia de anastomosis $(n=92)$ (Continuación)

\begin{tabular}{|c|c|c|c|c|c|}
\hline \multicolumn{3}{|c|}{ Variables cualitativas } & \multicolumn{2}{|c|}{ Dehiscencia de anastomosis } & \multirow{2}{*}{$\begin{array}{c}\mathrm{p}^{*} \\
\text { (OR; IC95\%) }\end{array}$} \\
\hline & & & \multirow{2}{*}{$\frac{\text { Sí }(\mathbf{n}=12)}{9(9.8 \%)}$} & \multirow{2}{*}{$\frac{\text { No }(\mathbf{n}=\mathbf{8 0})}{36(39.1 \%)}$} & \\
\hline & Anastomosis primaria & Sí & & & 0.05 \\
\hline & & No & $3(3.3 \%)$ & $44(47.8 \%)$ & $0.92-14.5)$ \\
\hline & Tipo de anastomosis & Manual & $12(13 \%)$ & $76(82.6 \%)$ & 0.60 \\
\hline & & Mecánica & $0(0 \%)$ & $4(4.3 \%)$ & $0.79-0.93)$ \\
\hline & Estoma protector o profiláctico & Sí & $3(3.3 \%)$ & $12(13.0 \%)$ & 0.30 \\
\hline & & No & $9(9.8 \%)$ & $68(73.9 \%)$ & $(1.8 ; 0.44-8.0)$ \\
\hline \multirow[t]{6}{*}{ Posoperatorio } & NPT & Sí & $5(5.4 \%)$ & $16(17.3 \% 9$ & 0.10 \\
\hline & & No & $7(7.6 \%)$ & $64(69.5 \%)$ & $0.80-10.1)$ \\
\hline & Pase a UCl & Sí & $1(1.1 \%)$ & $8(8.7 \%)$ & 0.66 \\
\hline & & No & $11(12: 0 \%)$ & $72(78.3 \%)$ & $0.93-1.1)$ \\
\hline & Mortalidad asociada al procedimiento. & Sí & $1(1.1 \%)$ & $6(6.5 \%)$ & 0.41 \\
\hline & & No & $11(12.9 \% 9$ & $74(80.4 \%)$ & .2) \\
\hline
\end{tabular}

ASA: Clasificación Asociación Americana de Anestesiología; IC95\%: intervalo de confianza del 95\%; IMC: índice de masa corporal; NPT: nutrición parenteral total; OR: odds ratio; UCl: unidad de cuidados intensivos.

*Prueba de ji al cuadrado.

†Antiinflamatorios no esteroideos, metformina, enalapril, pravastatina.

Tabla 5. Análisis de variables cuantitativas según la presencia de dehiscencia de anastomosis $(n=92)$

\begin{tabular}{lcccc}
\hline $\begin{array}{l}\text { Variables } \\
\text { cuantitativas }\end{array}$ & \multicolumn{2}{c}{$\begin{array}{c}\text { Dehiscencia de } \\
\text { anastomosis }\end{array}$} & $\begin{array}{c}\text { p } \\
\text { (IC95\%) }\end{array}$ \\
\cline { 2 - 3 } & $\begin{array}{c}\text { Sín } \\
(\mathbf{n}=\mathbf{1 2})\end{array}$ & $\begin{array}{c}\text { No } \\
(\mathbf{n}=\mathbf{8 0})\end{array}$ & \\
\hline Edad & $39.0 \pm 9.8$ & $43.9 \pm 16.9$ & $0.194(-15.15-4.9)$ \\
Albúmina sérica & $3.3 \pm 1.0$ & $3.4 \pm 0.8$ & $0.709(-8.19-0.6)$ \\
$\begin{array}{l}\text { Duración de la cirugía } \\
\text { (minutos) }\end{array}$ & $152.6 \pm 43$ & $179.0 \pm 67.3$ & $0.089(-57.1-4.3)$ \\
$\begin{array}{l}\text { Días de estancia } \\
\text { hospitalaria }\end{array}$ & $29.1 \pm 8.4$ & $11.3 \pm 1.2$ & $0.000(8.8-26.9)$ \\
$\begin{array}{l}\text { Inicio de dieta enteral } \\
\text { (días) }\end{array}$ & $3.9 \pm 2.7$ & $3.6 \pm 2.1$ & $0.23(-1.15-1.6)$ \\
\hline $\begin{array}{l}\text { IC95\%: intervalo de confianza del } 95 \% . \\
\text { *Pruebat de Student. }\end{array}$ & & & \\
\hline
\end{tabular}

necesario controlar el foco de infección, desmantelar la anastomosis previa y realizar un estoma derivativo ${ }^{20}$.

El inicio de la dieta enteral sí fue prolongado en pacientes con o sin dehiscencia, ya que el promedio fue de 3.7 días; aun cuando las guías de nutrición en cirugía indican recomendaciones fuertes en cuanto a que la dieta enteral no debe interrumpirse ${ }^{21}$. Entre las causas de falta de apego a la terapia nutricional en diversos centros hospitalarios, descritas por Robertson, et al. ${ }^{22}$ en 2018, predomina la falta de estandarización del método y de coordinación entre los servicios de cirugía y de nutrición en los hospitales para asegurar que se implemente esta práctica en aquellos pacientes que la requieran.

Los factores que se identifican ante la presencia de una dehiscencia de anastomosis se deben clasificar de acuerdo con el momento de la intervención quirúrgica (preoperatorio, transoperatorio y posoperatorio), con la finalidad de obtener información detallada que permita identificar puntos críticos de riesgo, así como implementar medidas preventivas según las características de cada paciente.

\section{Conclusiones}

Nuestro estudio muestra resultados comparables a los descritos en la literatura. Los factores de riesgo para el desarrollo de dehiscencia en nuestra población fueron la ingesta previa de medicamentos y la anastomosis primaria.

El manejo de las anastomosis intestinales se debe estandarizar de acuerdo con la evidencia disponible, y hay que implementar protocolos para la preparación previa de los pacientes que requerirán 
Cirugía y Cirujanos. 2021;89(2)

Tabla 6. Análisis univariado asociado al sitio anatómico de la anastomosis $(n=92)$

\begin{tabular}{|c|c|c|c|c|c|}
\hline \multicolumn{3}{|c|}{ Variables cualitativas } & \multicolumn{2}{|c|}{ Sitio anatómico } & \multirow{2}{*}{$\begin{array}{c}p^{*} \\
\text { (OR; IC95\%) }\end{array}$} \\
\hline & & & \multirow{2}{*}{$\begin{array}{c}\begin{array}{c}\text { Intestino delgado } \\
(\mathbf{n}=\mathbf{2 8})\end{array} \\
23(25.0 \%)\end{array}$} & \multirow{2}{*}{$\begin{array}{c}\begin{array}{c}\text { Intestino grueso } \\
(\mathbf{n}=\mathbf{6 4})\end{array} \\
47(51.1 \%)\end{array}$} & \\
\hline Preoperatorio & Sexo & Masculino & & & \multirow{2}{*}{$\begin{array}{c}0.43 \\
(1.66 ; 0.54-5.0)\end{array}$} \\
\hline & & Femenino & $5(5.4 \%)$ & $17(18.5 \%)$ & \\
\hline & \multirow[t]{2}{*}{ Tabaquismo } & Sí & $17(18.5 \%)$ & $44(47.8 \%)$ & \multirow{2}{*}{$\begin{array}{c}0.48 \\
(0.72 ; 0.27-1.77)\end{array}$} \\
\hline & & No & $11(12.0 \%)$ & $20(21.7 \%)$ & \\
\hline & \multirow[t]{2}{*}{ Ingesta previa de medicamentos ${ }^{\dagger}$} & Sí & $5(5.4 \%)$ & $24(26.1 \%)$ & \multirow{2}{*}{$\begin{array}{c}0.050 \\
(1.3 ; 1.05-1.67)\end{array}$} \\
\hline & & No & $23(25.0 \%)$ & $40(43.5 \%)$ & \\
\hline & \multirow[t]{2}{*}{ Comorbilidad } & Sí & $6(6.5 \%)$ & $22(23.9 \%)$ & \multirow{2}{*}{$\begin{array}{c}0.16 \\
(0.52 ; 0.18-1.4)\end{array}$} \\
\hline & & No & $22(23.9 \%)$ & $42(45.7 \%)$ & \\
\hline & \multirow[t]{4}{*}{ IMC } & Desnutrido & $2(2.2 \%)$ & $2(2.2 \%)$ & \multirow{4}{*}{$\begin{array}{c}0.13 \\
(1.3 ; 0.62-0.19)\end{array}$} \\
\hline & & Normal & $11(12.0 \%)$ & $26(28.3 \%)$ & \\
\hline & & Sobrepeso & $6(6.5 \%)$ & $26(28.3 \%)$ & \\
\hline & & Obesidad & $9(9.8 \%)$ & $26(28.3 \%)$ & \\
\hline & \multirow[t]{2}{*}{ Preparación intestinal } & Sí & $7(7.6 \%)$ & $26(28.3 \%)$ & \multirow{2}{*}{$\begin{array}{c}0.11 \\
(0.48 ; 0.18-1.3)\end{array}$} \\
\hline & & No & $21(22.8 \%)$ & $38(41.3 \%)$ & \\
\hline & \multirow[t]{2}{*}{ Profilaxis antibiótica } & Sí & $11(12.0 \%)$ & $29(31.5 \%)$ & \multirow{2}{*}{$\begin{array}{c}0.38 \\
(0.78 ; 0.31-1.9)\end{array}$} \\
\hline & & No & $17(18.5 \%)$ & $35(38.5 \%)$ & \\
\hline & \multirow[t]{3}{*}{ Estado físico ASA } & 1 & $7(7.6 \%)$ & $22(23.9 \%)$ & \multirow{3}{*}{$\begin{array}{c}0.33 \\
(0.66 ; 0.00-0.69)\end{array}$} \\
\hline & & $\|$ & $11(12.0 \%)$ & $32(34.8 \%)$ & \\
\hline & & III & $10(10.9 \%)$ & $10(10.9 \%)$ & \\
\hline & \multirow[t]{2}{*}{ Tipo de cirugía } & Electiva & $15(16.3 \%)$ & $52(56.5 \%)$ & \multirow{2}{*}{$\begin{array}{c}0.006 \\
(1.6 ; 1.05-2.48)\end{array}$} \\
\hline & & Urgencia & $13(14.1 \%)$ & $1213.0 \%)$ & \\
\hline \multirow[t]{18}{*}{ Transoperatorio } & \multirow[t]{2}{*}{ Sepsis abdominal } & Sí & $13(14.1 \%)$ & $1213.0 \%)$ & \multirow{2}{*}{$\begin{array}{c}0.006 \\
(3.7 ; 1.42-9.93)\end{array}$} \\
\hline & & No & $15(16.3 \%)$ & $52(56.5 \%)$ & \\
\hline & \multirow[t]{5}{*}{ Transfusiones transoperatorias } & Ninguna & $20(21.7 \%)$ & $53(57.6 \%)$ & 0.65 \\
\hline & & 1 & $3(3.3 \%)$ & $7(7.6 \%)$ & $0.015-0.116)$ \\
\hline & & 2 & $3(3.3 \%)$ & $4(4.3 \%)$ & \\
\hline & & 3 & 1 & 0 & \\
\hline & & 5 & 1 & 0 & \\
\hline & Drenajes & Sí & $21(22.8 \%)$ & $48(52.2 \%)$ & 0.20 \\
\hline & & No & $7(7.6 \%)$ & $16(17.4 \%)$ & $(1.0 ; 0.35-2.78)$ \\
\hline & Causa de la cirugía & Benigna & $18(19.6 \%)$ & $49(53.3 \%)$ & 0.163 \\
\hline & & Maligna & $6(6.5 \%)$ & $10(10.9 \%)$ & $(1.2 ; 0.88-0.23)$ \\
\hline & & Traumatismo & $4(4.3 \%)$ & $5(5.4 \%)$ & \\
\hline & Anastomosis primaria & Sí & $16(17.4 \%)$ & $29(31.5 \%)$ & 0.20 \\
\hline & & No & $12(13.0 \%)$ & $35(38.5 \%)$ & $\begin{array}{c}(0.86 \\
0.65-3.94)\end{array}$ \\
\hline & Tipo de anastomosis & Manual & $28(30.4 \%)$ & $60(65.2 \%)$ & 0.22 \\
\hline & & Mecánica & $0(0 \%)$ & $4(4.3 \%)$ & 0.59-0.78) \\
\hline & Estoma protector o profiláctico & Sí & $4(4.3 \%)$ & $11(12.0 \%)$ & 0.49 \\
\hline & & No & $24(26.1 \%)$ & 53 (57.6\%) & $(0.80 ; 0.23-2.78)$ \\
\hline
\end{tabular}


Tabla 6. Análisis univariado asociado al sitio anatómico de la anastomosis $(n=92)$ (Continuación)

\begin{tabular}{|c|c|c|c|c|c|}
\hline \multicolumn{3}{|c|}{ Variables cualitativas } & \multicolumn{2}{|c|}{ Sitio anatómico } & \multirow{2}{*}{$\begin{array}{c}\mathrm{p}^{\star} \\
\text { (OR; IC95\%) }\end{array}$} \\
\hline & & & \multirow{2}{*}{$\begin{array}{c}\begin{array}{c}\text { Intestino delgado } \\
(\mathbf{n}=\mathbf{2 8})\end{array} \\
10(10.9 \%)\end{array}$} & \multirow{2}{*}{$\begin{array}{c}\begin{array}{c}\text { Intestino grueso } \\
(\mathbf{n}=64)\end{array} \\
11(12.0 \%)\end{array}$} & \\
\hline \multirow[t]{8}{*}{ Posoperatorio } & \multirow[t]{2}{*}{ NPT } & Sí & & & \multirow{2}{*}{$\begin{array}{c}0.049 \\
(2.6 ; 0.97-7.34)\end{array}$} \\
\hline & & No & $18(19.6 \%)$ & $53(57.6 \%)$ & \\
\hline & \multirow[t]{2}{*}{ Dehiscencia } & Sí & $4(4.3 \%)$ & $8(8.7 \%)$ & \multirow{2}{*}{$\begin{array}{c}0.52 \\
(0.92 \\
0.623-1.45)\end{array}$} \\
\hline & & No & $24(26.1 \%)$ & $56(60.9 \%)$ & \\
\hline & \multirow[t]{2}{*}{ Pase a UCl } & Sí & $4(4.3 \%)$ & $5(5.4 \%)$ & \multirow{2}{*}{$\begin{array}{c}0.27 \\
(0.78 \\
0.42-1.42)\end{array}$} \\
\hline & & No & $24(26.1 \%)$ & $59(64.1 \%)$ & \\
\hline & \multirow[t]{2}{*}{$\begin{array}{l}\text { Mortalidad asociada al } \\
\text { procedimiento }\end{array}$} & Sí & $2(2.2 \%)$ & $5(5.4 \%)$ & \multirow{2}{*}{$\begin{array}{c}0.64 \\
(0.90 \\
0.165-4.98)\end{array}$} \\
\hline & & No & $26(28.3 \%)$ & $59(64.1 \%)$ & \\
\hline
\end{tabular}

ASA: Clasificación Asociación Americana de Anestesiología; IC95\%: intervalo de confianza del 95\%; IMC: índice de masa corporal; NPT: nutrición parenteral total; OR: odds ratio;

UCl: unidad de cuidados intensivos.

*Prueba de ji al cuadrado.

${ }^{\dagger}$ Antiinflamatorios no esteroideos, metformina, enalapril, pravastatina.

anastomosis intestinal, tanto en cirugía electiva como de urgencia.

\section{Conflicto de intereses}

Los autores declaran que no existe conflicto de intereses para el presente artículo.

\section{Responsabilidades éticas}

Protección de personas y animales. Los autores declaran que para esta investigación no se han realizado experimentos en seres humanos ni en animales.

Confidencialidad de los datos. Los autores declaran que han seguido los protocolos de su centro de trabajo sobre la publicación de datos de pacientes.

Derecho a la privacidad y consentimiento informado. Los autores declaran que en este artículo no aparecen datos de pacientes.

\section{Bibliografía}

1. Matsuda A, Miyashita M, Matsumoto S, Nobuyuki, Takahashi M, Uchida Y. Isoperistaltic versus antiperistaltic stapled side-to-side anastomosis for colon cancer surgery: a randomized controlled trial. J Surg Res. 2015;196:107-12.

2. Daams F, Luyer M, Lange J. Colorectal anastomotic leakage: aspects of prevention, detection and treatment. World $\mathrm{J}$ Gastroenterol. 2013;19:2293-7.

3. Dekker J, Liefers G, Mol van Otterloo J, Putter H, Tollenaar R. Predicting the risk of anastomotic leakage in left-sided colorectal surgery using a colon leakage score. J Surg Res. 2011;166:327-e34.
4. Hyman N. Managing anastomotic leaks from intestinal anastomoses. Surgeon. 2009;7:31-5

5. Elemen L, Sanmurat N, Ayik B, Aydin S, Uzun, H. Is the use of cyanocrylate in intestinal anastomosis a good and reliable alternative? J Pediatr Surg. 2009;44:581-6.

6. Hong Ho Y, Mohamed A, Tawfik Ashour T. Techniques for colorectal anastomosis. World J Gastroenterol. 2010;16:1610-21.

7. Buchs NC, Gervaz P, Secic M, Bucher P, Mugnier-Konrad B, Morel P. Incidence, consequences, and risk factors for anastomotic dehiscence after colorectal surgery: a prospective monocentric study. Int J Colorectal Dis. 2008;23:265-70.

8. Shiomi A, Ito M, Maeda K, Kinugasa Y, Ota M, Yamaue H, et al. Effects of a diverting stoma on symptomatic anastomotic leakage after low anterior resection for rectal cancer: a propensity score matching analysis of 1014 consecutive patients. J Am Coll Surg. 2015;220:186-94.

9. Lipska M, Bissett I, Parry B, Merrie A. Anastomotic leakage after lower gastrointestinal anastomosis: men are at a higher risk. ANZ J Surg. 2006;76:579-85.

10. Bruce J, Krukowski Z, Al-Khairy G, Russell E, Park K. Systematic review of the definition and measurement of anastomotic leak after gastrointestinal surgery. Br J Surg. 2001;88:1157-68.

11. Alves A, Panis $Y$, Trancart D, Regimbeau J, Pocard M, Valleur P. Factors associated with clinically significant anastomotic leakage after large bowel resection: multivariate analysis of 707 patients. World J Surg. 2002;18:499-502.

12. Morse B, Simpson J, Jones Y, Johnson B, Knott B, Kotrady J. Determination of independent predictive factors for anastomotic leak: analysis of 682 intestinal anastomoses. Am J Surg. 2013;260:950-6.

13. Rojas-Machado S, Romero M, Arroyo A, Rojas-Machado A, López J, Calpena R. Anastomotic leak in colorectal cancer surgery. Development of a diagnostic index (DIACOLE). Inter J Surg. 2016;39:92-8.

14. Muñoz N, Rodríguez M, Pérez-Castila A, Campaña N, Campaña G. Evaluation of the risk factors associated with anastomotic dehiscence in colorectal surgery. Multivariate analysis of 748 patients. Rev Cir. 2019;71:136-44.

15. Iversen $\mathrm{H}$, Ahlberg $\mathrm{M}$, Lindqvist $\mathrm{M}$, Buchli $\mathrm{C}$. Changes in clinical practice reduce the rate of anastomotic leakage after colorectal resections. World J Surg. 2018;42:2234-41.

16. Kosuge M, Eto K, Hashizume R, Takeda M, Tomori K, Mitsouri N, et al. Wich is the safer anastomotic method for colon surgery? Ten-year results. In vivo. 2017:31:683-7.

17. Beyer-Berjot L, Slim K. Colorectal surgery and preoperative bowel preparation: aren't we drawing hasty conclusions? Colorectal Dis. 2018;20:955-8.

18. Guerra F, Giuliani G, Coletta D, Marcello Boni M, Rondelli F, Pietro Bianch $\mathrm{P}$, et al. A meta-analysis of randomized controlled trials on the use of suction drains following rectal surgery. Dig Surg. 2018;35:482-90. 
Cirugía y Cirujanos. 2021;89(2)

19. Hallam S, Mothe BS, Tirumulaju RM. Hartmann's procedure, reversal and rate of stoma-free survival. Ann R Coll Surg Engl. 2018;100:301-7.

20. Thomas M, Margolin D. Management of colorectal anastomotic leak. Clin Colon Rectal Surg. 2016;29:138-44.
21. Weimann A, Braga M, Carli F, Higashiguhi T, Hübner M, Klek S. ESPEN guideline: clinical nutrition in surgery. Clin Nutr. 2017;36:623-50.

22. Robertson TR, Eldridge NE, Rattray ME, Roberts SJ, Desbrow B, Marshall AP, et al. Early oral feeding after colorectal surgery: a mixed methods study of knowledge translation. Nutr Diet. 2018;75:345-52. 